\title{
A56 THE TYPE 1 INTERFERON SIGNATURE: FACTS, FADS AND FALLACIES
}

Thomas Häupl, ${ }^{1}$ Robert Biesen, ${ }^{1}$ Biljana Smiljanovic, ${ }^{2}$ Joachim R Grün, ${ }^{2}$ Janine Weix, ${ }^{3}$ Bruno Stuhlmüller, ${ }^{1}$ Peter M Villiger, ${ }^{3}$ Gerd-R Burmester, ${ }^{1}$ Andreas Radbruch, ${ }^{2}$ Andreas Grützkau ${ }^{2}{ }^{1}$ Department of Rheumatology and Clinical Immunology, Charité Universitätsmedizin, Berlin, Germany; ${ }^{2}$ German Arthritis Research Center, Berlin, Germany; ${ }^{3}$ Department of Rheumatology and Clinical Immunology, Inselspital, Bern, Switzerland

\subsection{6/ard.2010.148965.26}

Background and objectives The type 1 interferon signature (IFN1) has been reported in systemic lupus erythematosus (SLE) and subsequently in many other diseases. It is deduced from the expression of a group of genes known as downstream events after IFN1 stimulation. Involving certain toll-like receptors and being related especially to an antiviral immune response, interpretation of these findings is difficult, in particular if such signatures are reported in bacterial infections as well. Therefore, the authors aimed for a better interpretation by comparing qualities and quantities of the IFN1 signature after stimulation and in various diseases.

Materials and methods Transcriptomes of chronic inflammatory diseases, infections and defined stimulation experiments with IFN1 and other cytokines, which were generated by the authors or retrieved from the Gene Expression Omnibus were systematically investigated using the BioRetis analysis platform for primary analysis and Genesis for comparative visualisation.

Results A typical IFN1 signature was identified by screening for differentially expressed genes before and after IFN1 treatment in patients or in vitro stimulation of immune cells with IFN1 compared to other cytokines. The pattern of these genes was compared with the pattern in transcriptomes from patients with different rheumatic diseases including rheumatoid arthritis (RA), spondyloarthritis, SLE, Sjögren's Syndrome, Myositis, Scleroderma and juvenile rheumatic diseases as well as with infectious diseases. Leading candidates related to IFN1 were IFIT3, IFI44L, IFI35, IFI44, MX1, MX2, OAS1, OAS2, OAS3 or SIGLEC1 already known from previous studies. These genes were characteristic for viral infections, SLE and other connective tissue diseases. Bacterial infections were positive only in few exceptions, suggesting co-infection with viruses. When observed in arthritic conditions, the diagnosis of RA was challenged. When comparing signatures induced in vitro, a much broader range of immune reactions was observed in early stages with substantial overlap between different cytokines. However, magnitude of change was still an indicator for specificity, suggesting that specific triggers and responses are always associated with

additional more or less unspecific activation patterns. This may be a general rule to enable subsequently the development of a higher complexity of the immune response whenever necessary.

Conclusions In summary, the IFN1 signature is certainly a prominent observation in the current literature. However, definitions for the IFN1 response have to be applied carefully in order to avoid misinterpretations by overestimation of minor or negligible amounts. Therefore, analysis should include comparisons with typical positive IFN1 signatures as reference and if possible with changes in other pathways relevant for differential diagnosis. 